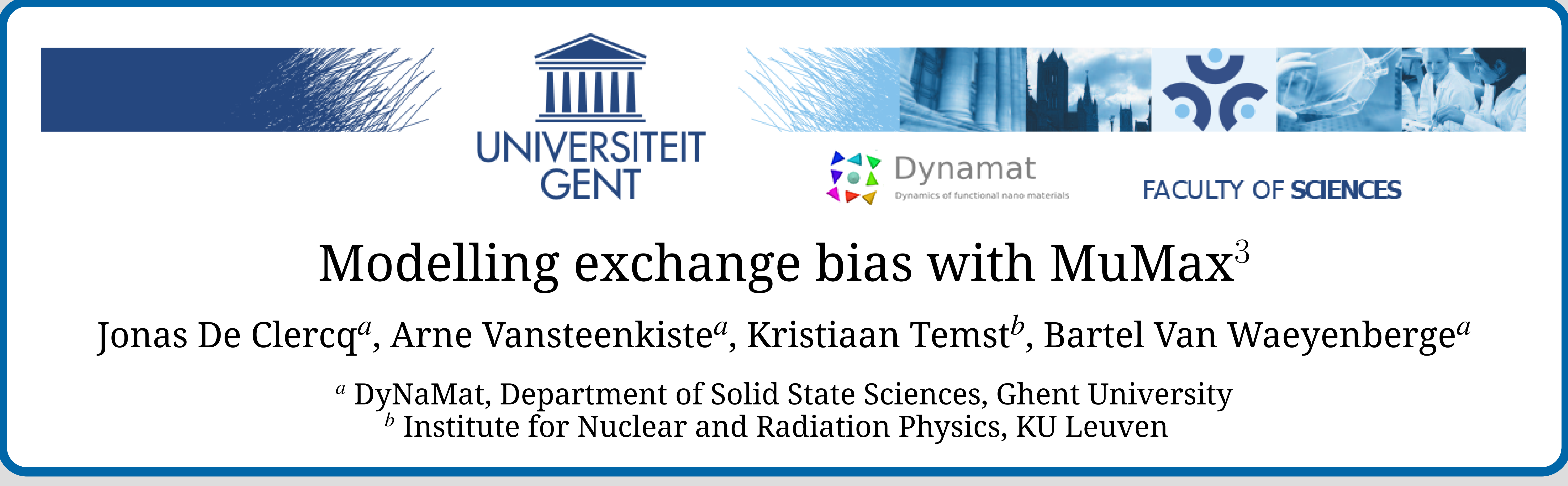

\title{
Exchange bias
}

When cooling a ferromagnetic / antiferromagnetic bilayer in an external magnetic field below the Néel temperature $T_{N}$, an unidirectional shift of the hysteresis loop is found due to the coupling between both layers. This effect is used in GMR read heads under the form of spin valves.

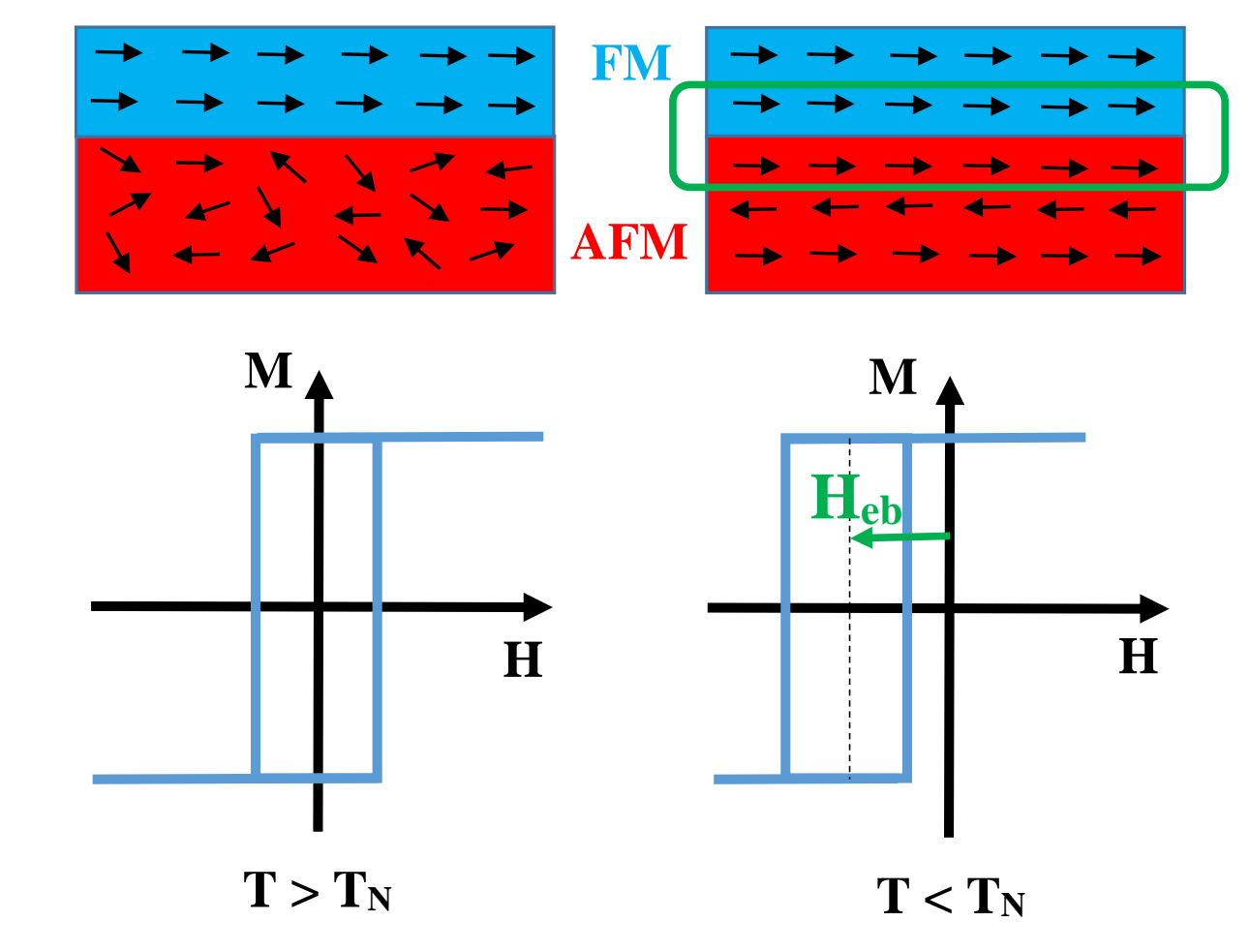

exchange biased hysteresis loop

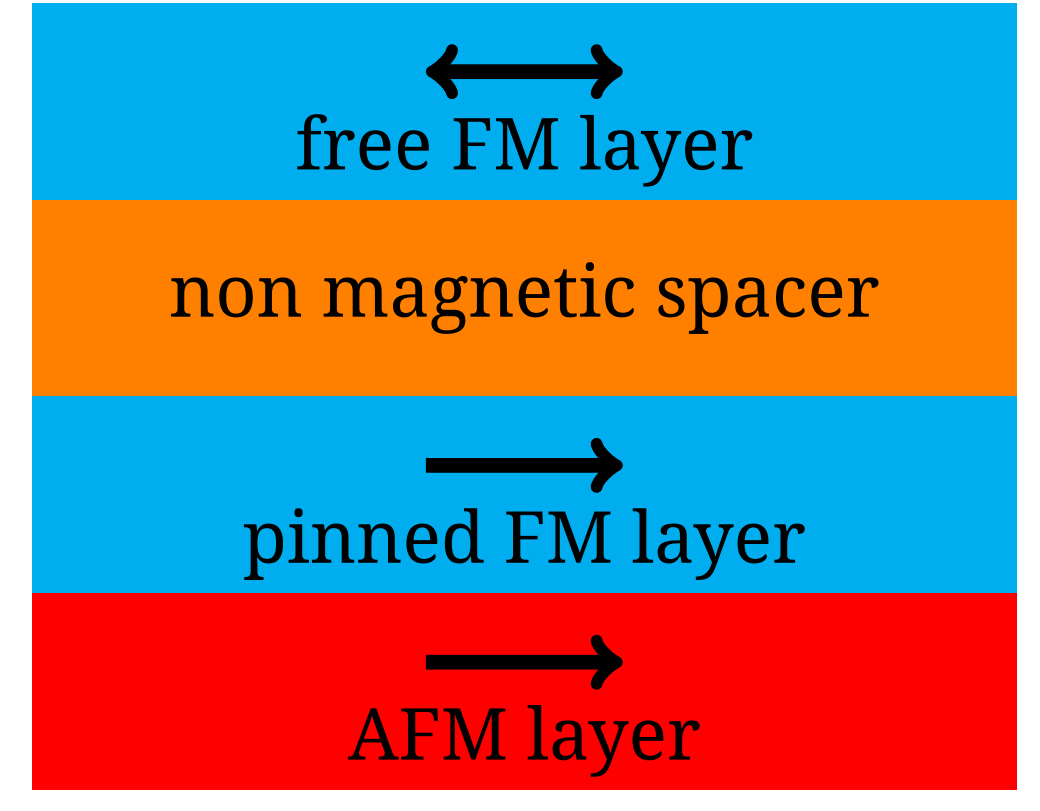

spin valve

In most polycrystalline stacks also a training effect can be seen, i.e. the coercivity and bias field decrease for an increasing number of hysteresis cycles $n$. For $n=1$, the athermal component of the training effect contains the largest contribution.

\section{Objective}

We proof, by reproducing experimental data[1] for an exchange biased $\mathrm{Co}(30 \mathrm{~nm}) / \mathrm{CoO}(3 \mathrm{~nm})$ bilayer, that we can include these 2 effects in $\operatorname{MuMax}^{3}$ by considering the presence of pinned and rotatable grains in the AFM layer. We also demonstrate there can be an asymmetry in the reversal mechanism between the descending branch for $n=1$ and further hysteresis loops.

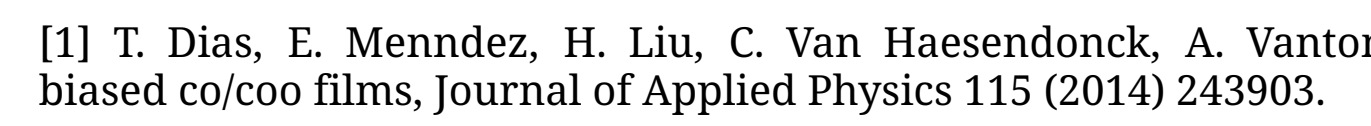

\section{Solving micromagnetism with $\mathrm{MuMax}^{3}$}

In micromagnetism the evolution of the magnetisation $\vec{M}(\vec{r}, t)$ in an effective field $\vec{H}_{e f f}$ is determined by the Landau - Lifshitz equation

$$
\left(1+\alpha^{2}\right) \frac{\partial \vec{M}}{\partial t}=-\gamma \vec{M} \times \vec{H}_{e f f}-\left(\frac{\alpha \gamma}{M_{s}}\right) \vec{M} \times\left(\vec{M} \times \vec{H}_{e f f}\right)
$$

with $\alpha$ the dimensionless damping constant and $\gamma$ the gyromagnetic ratio. $\mathbf{M u M a x}^{3}$ [2], which is a GPU - accelerated open source micromagnetic simulation program, allows us to solve this equation for a ferromagnetic system by using a finite difference discretisation.

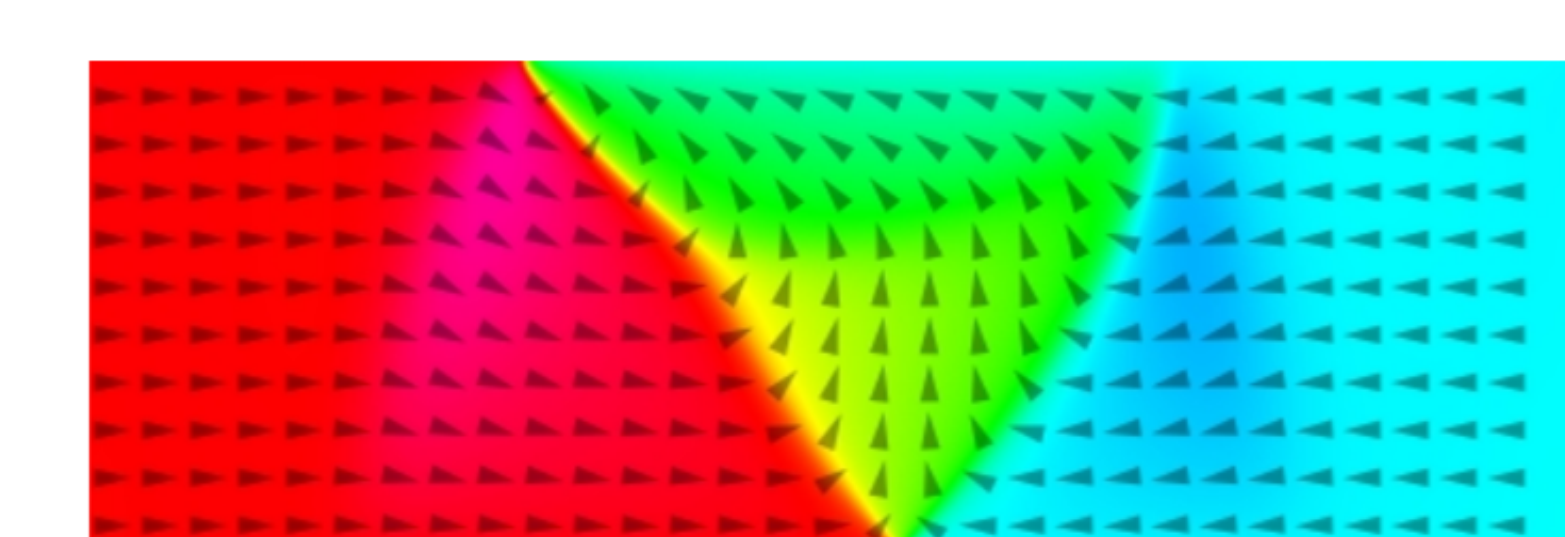
Néel domain wall in FM nanowire magnetic vortex

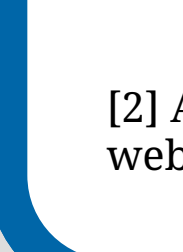

$$
\text { [2] }
$$

\section{Micromagnetic model}

The polycrystalline $\mathrm{Co}$ and $\mathrm{CoO}$ layers are each divided into grains with an average grain size of $12 \mathrm{~nm}$ using a Voronoi tessellation. The anisotropy axes of the FM grains are distributed around the field cooling direction according to a normal distribution with a standard deviation of $10^{\circ}$.

The AFM grains are divided into 2 types, pinned and rotatable, according to a ratio of 3:7 respectively. Their anisotropy axes are randomly distributed in plane. No Zeeman or demagnetization energy was taken into account for the AFM layer.

\section{Unidirectional shift}

Pinned AFM grains, which have a high anisotropy constant and so are almost frozen, cause the unidirectional shift of the hysteresis loop due to their coupling with the FM layer. Using an exchange stiffness of $A_{\text {pin }}=6.9 \times 10^{-12} \mathrm{~J} / \mathrm{m}$ and $A_{\text {rot }}=1.1 \times 10^{-11} \mathrm{~J} / \mathrm{m}$ at the interface, we find that our bias field and coercivity agrees well with the experimental data.

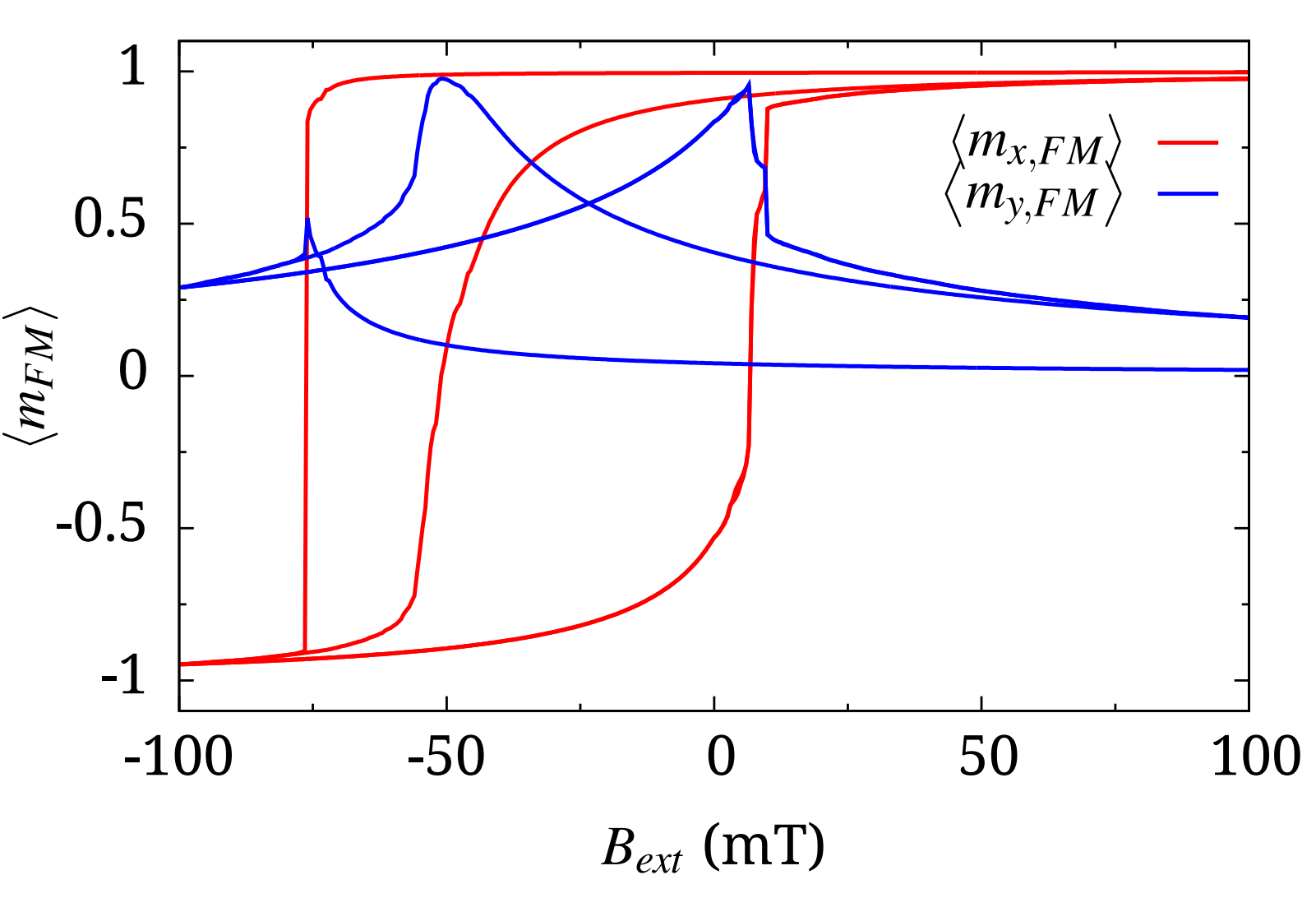

hysteresis loop of the FM layer

\section{Athermal training effect}

After field cooling, the magnetization of the AFM grains is randomly distributed in the field cooling direction. Rotatable AFM grains have a low anisotropy constant $\left(K_{U}=2.0 \times 10^{6} \mathrm{~J} / \mathrm{m}^{3}\right)$ and so rotate together with the FM layer during the hysteresis loop. Especially those grains with an anisotropy axis almost perpendicular to the field cooling direction contribute to the athermal training effect as explained below.
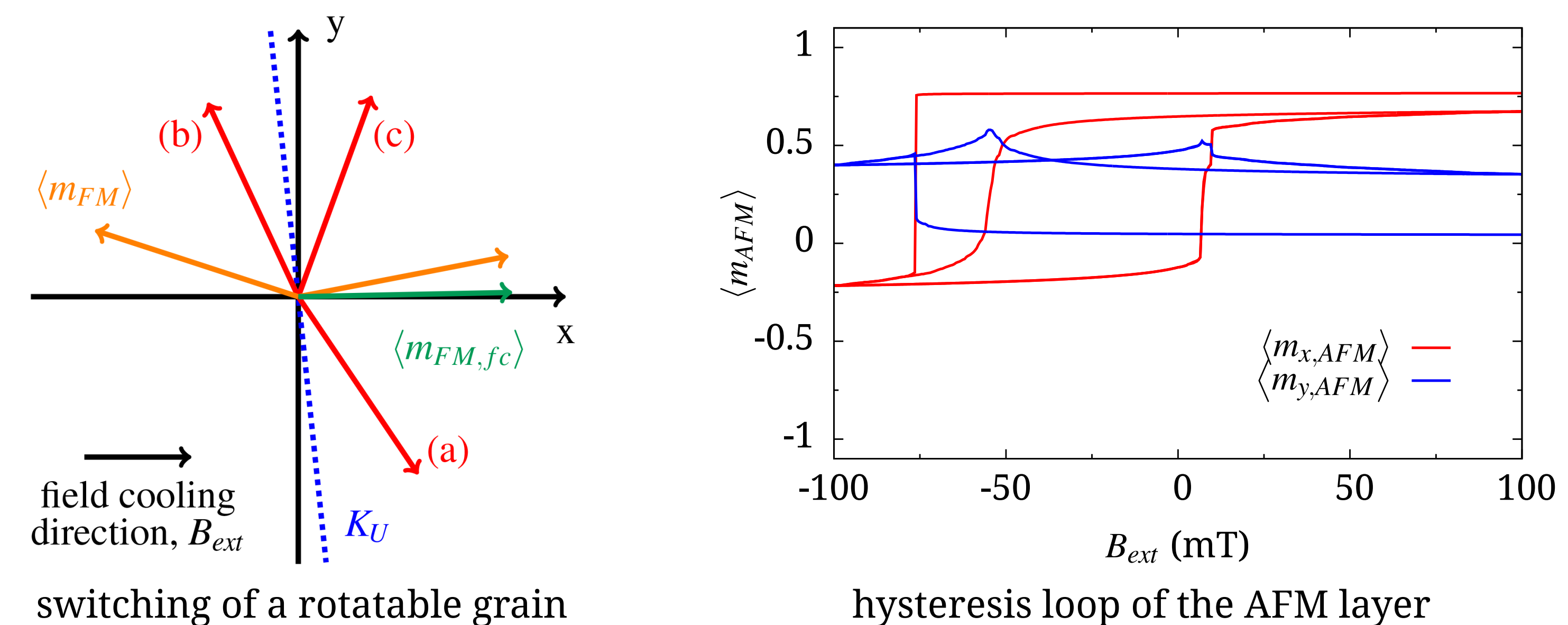

hysteresis loop of the AFM layer

Suppose the magnetisation of such a rotatable AFM grain is at position (a) after field cooling and that the FM rotates coherently. If the FM has a small initial positive $\left\langle m_{y, F M}\right\rangle$ component, the AFM grain will rotate counterclockwise in the descending branch of the first hysteresis loop and jump irreversibly towards position (b). After the first hysteresis cycle, the AFM grain relaxes towards position (c) and so does not return to its initial position. This results in a non closed AFM hysteresis loop for $n=1$.

For $\mathbf{n}>\mathbf{1}$ the grain reversibly switches between (b) and (c). As now $\left\langle m_{y, A F M}\right\rangle>0$, the AFM applies a net torque on the FM layer which results in a lower coercivity and bias field. For FM domains in which $\left\langle m_{y, F M}\right\rangle<0$, the reasoning is similar.
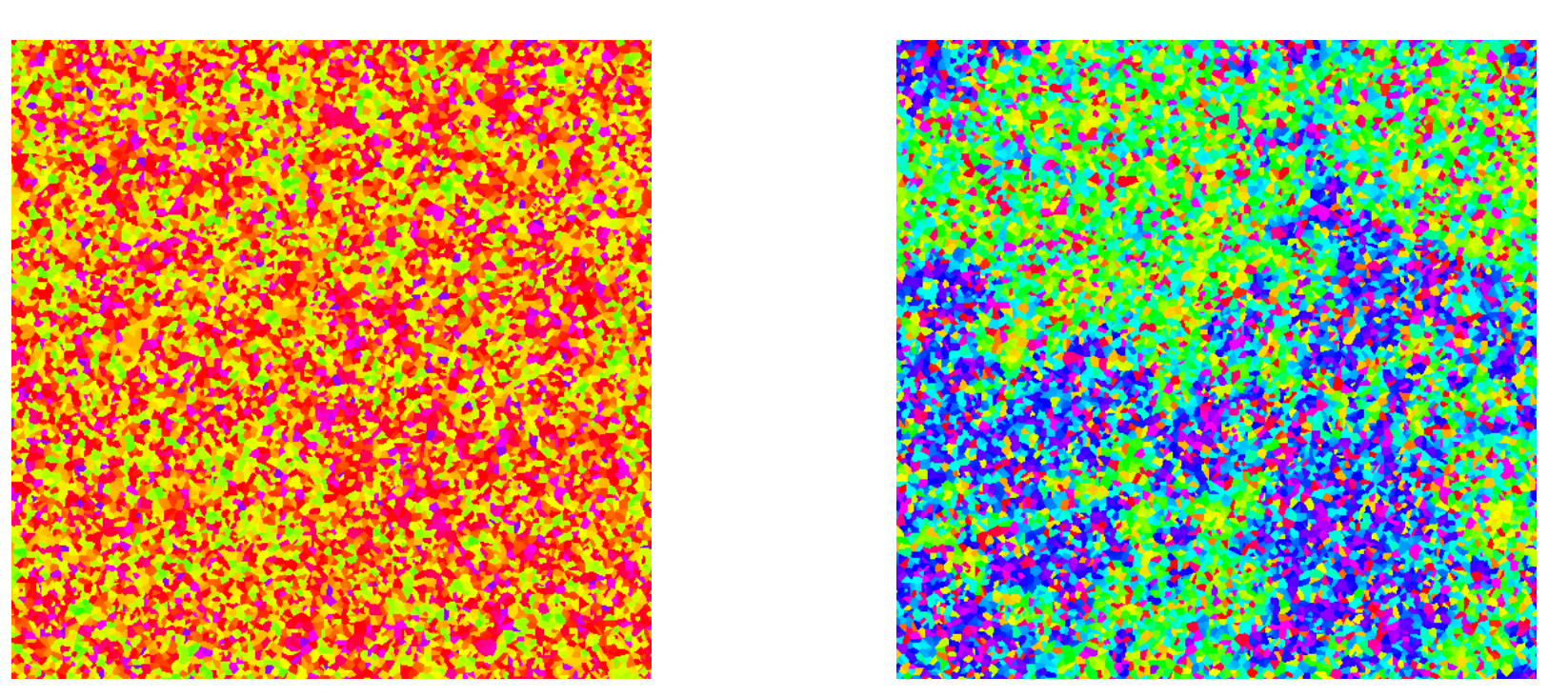

field cooled

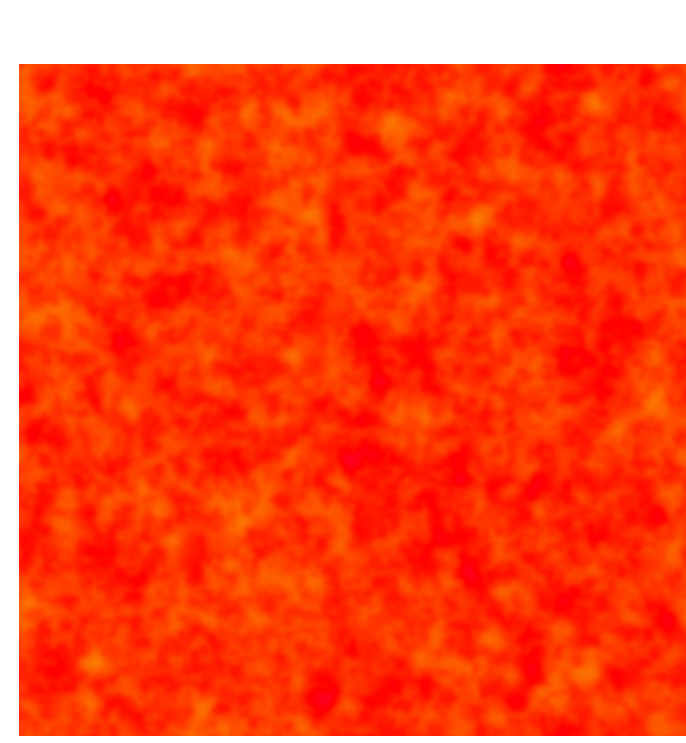

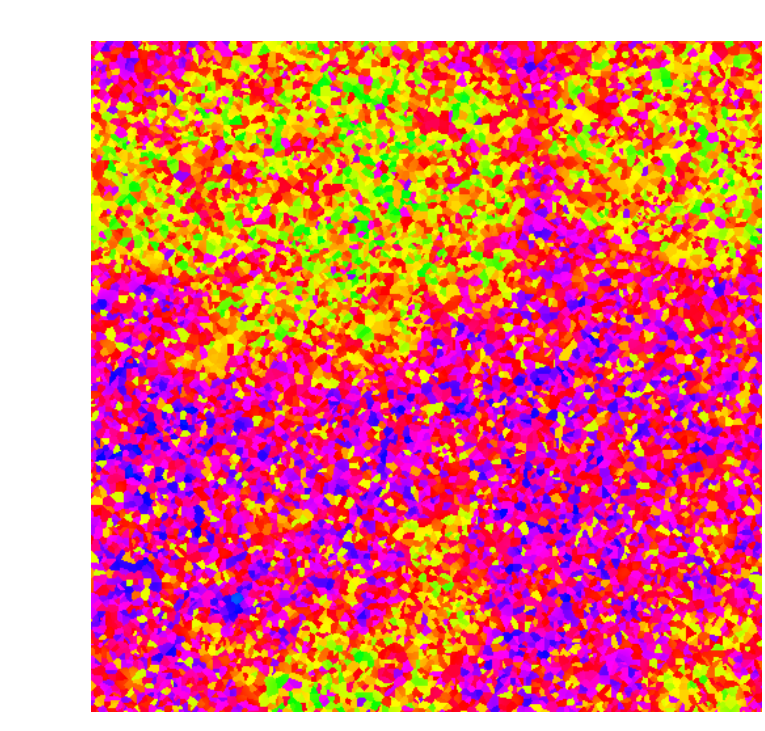

$\mathrm{n}=2, B_{\text {ext }}=-45 \mathrm{mT}$

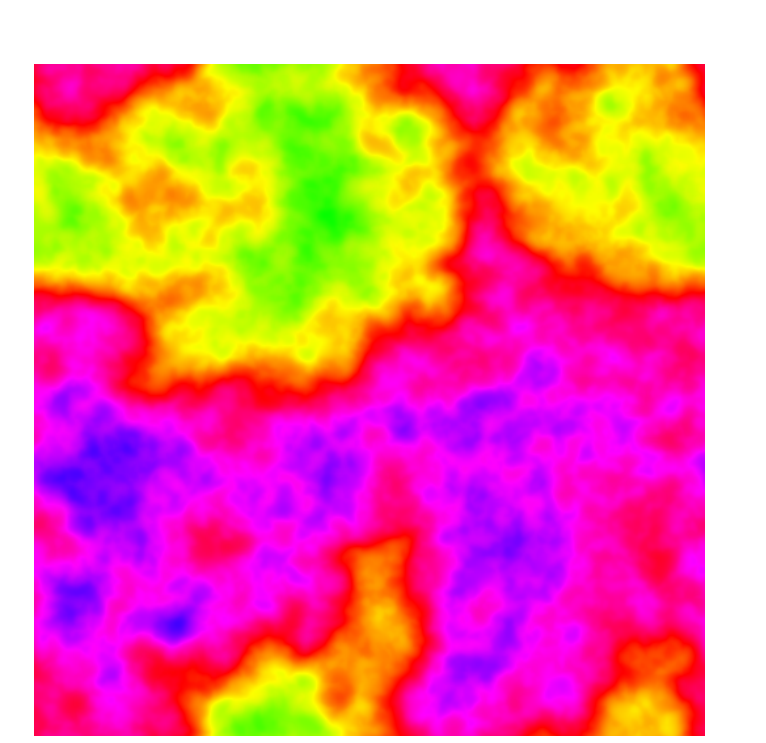

top row: AFM layer, bottom row: corresponding FM layer 